\title{
FORMATION OF GLOBULAR CLUSTERS IN ATOMIC-COOLING HALOS VIA RAPID GAS CONDENSATION AND FRAGMENTATION DURING THE EPOCH OF REIONIZATION
}

\author{
Taysun Kimm ${ }^{1,4}$, Renyue Cen ${ }^{1}$, Joakim Rosdahl ${ }^{2}$, and Sukyoung K. Yi ${ }^{3}$ \\ ${ }^{1}$ Princeton University Observatory, Peyton Hall, 4 Ivy Lane, Princeton, NJ 08544-1001, USA; tkimm@ast.cam.ac.uk \\ ${ }^{2}$ Leiden Observatory, Leiden University, P.O. Box 9513, 2300 RA, Leiden, The Netherlands \\ ${ }^{3}$ Department of Astronomy and Yonsei University Observatory, Yonsei University, Seoul 120-749, Korea \\ Received 2015 October 8; accepted 2016 April 5; published 2016 May 23
}

\begin{abstract}
We investigate the formation of metal-poor globular clusters (GCs) at the center of two dark matter halos with $M_{\text {halo }} \sim 4 \times 10^{7} M_{\odot}$ at $z>10$ using cosmological radiation-hydrodynamics simulations. We find that very compact $(\lesssim 1 \mathrm{pc})$ and massive $\left(\sim 6 \times 10^{5} M_{\odot}\right)$ clusters form rapidly when pristine gas collapses isothermally with the aid of efficient Ly $\alpha$ emission during the transition from molecular-cooling halos to atomic-cooling halos. Because the local free-fall time of dense star-forming gas is very short ( $\ll 1 \mathrm{Myr}$ ), a large fraction of the collapsed gas is turned into stars before stellar feedback processes blow out the gas and shut down star formation. Although the early stage of star formation is limited to a small region of the central star-forming disk, we find that the disk quickly fragments due to metal enrichment from supernovae. Sub-clusters formed in the fragmented clouds eventually merge with the main cluster at the center. The simulated clusters closely resemble the local GCs in mass and size but show a metallicity spread that is much wider than found in the local GCs. We discuss a role of preenrichment by Pop III and II stars as a potential solution to the latter issue. Although not without shortcomings, it is encouraging that a naive blind (not tuned) cosmological simulation presents a possible channel for the formation of at least some massive GCs.
\end{abstract}

Key words: galaxies: high-redshift - globular clusters: general

\section{INTRODUCTION}

Numerical simulations show that a variety of systems can emerge during the hierarchical build-up of small structures in an LCDM cosmology. Disk-dominated galaxies are formed by the coherent accretion of cold gas in conjunction with the removal of low-angular momentum gas via effective stellar feedback (Governato et al. 2007; Pichon et al. 2011; Dubois et al. 2014), while violent relaxation during galaxy mergers/ interactions leads to the formation of a massive bulge (e.g., Toomre \& Toomre 1972; Naab et al. 2006). In small halos with $M_{\text {vir }} \lesssim 10^{10} M_{\odot}$, supernova (SN) explosions seem to be able to alter the gravitational potential non-adiabatically by blowing out a significant amount of gas, producing diffuse dwarf galaxies (Pontzen \& Governato 2012). However, how dense structures, such as globular clusters (GCs), form in a cosmological context remains an interesting puzzle.

The formation of GCs is usually associated with high-pressure regions $\left(P / k_{B} \gtrsim 10^{7} \mathrm{~cm}^{-3} \mathrm{~K}\right.$, Elmegreen \& Efremov 1997), owing to their compact nature. This implies that the average density of the GC-forming, isothermal gas cores is very high $\left(n_{\mathrm{H}} \sim 10^{5}-10^{6} \mathrm{~cm}^{-3}\right)$, given the typical temperature of star-forming regions $(10-100 \mathrm{~K})$. Because the free-fall timescale of these clouds $\left(t_{\mathrm{ff}} \sim 0.1 \mathrm{Myr}\right)$ is an order of magnitude smaller than the typical dispersal timescale due to ionizing radiation ( $\sim 1-2 \mathrm{Myr}$, Walch et al. 2012; Dale et al. 2014; Sales et al. 2014) or a SN explosion (>3 Myr), the gas in the high-pressure regions can be efficiently converted into stars, although star formation itself is a slow process on galactic scales (Kennicutt 1998). An important difference between the GC formation sites and normal star-forming regions is that a large amount of gas needs to be accumulated

\footnotetext{
${ }^{4}$ Currently at the Kavli Institute for Cosmology and Institute of Astronomy, Madingley Road, Cambridge CB3 OHA, UK.
}

and collapse on a short timescale in the former case. If the gas accretion onto the cloud occurs very slowly compared to star formation, only a small fraction of the gas would achieve the high pressure and it may not form a typical, massive GC with $\sim 2 \times 10^{5} M_{\odot}$ before stellar feedback suppresses star formation. In this case, subsequent gas accretion after the initial burst of star formation is likely to lead to extended star formation histories, as in dwarf galaxies (e.g., Tolstoy et al. 2009).

Interacting galaxies or gas-rich, clumpy disks in high- $z$ galaxies are thus good candidates hosting such high-pressure regions. During a galaxy merger, gas is funneled rapidly to the central regions due to gravitational torques, triggering starbursts (Mihos \& Hernquist 1996). Tidal arms are also fragmented and create gas clumps, providing a favorable condition for cluster formation (e.g., Teyssier et al. 2010; Renaud et al. 2015). Indeed, young, metal-rich star clusters are often observed in nearby interacting galaxies (e.g., Whitmore \& Schweizer 1995; Schweizer et al. 1996; Bastian et al. 2009). Massive clumps with $\sim 10^{8}-10^{9} M_{\odot}$, observed in gas-rich disks at high redshifts (Genzel et al. 2008; Elmegreen et al. 2009; Swinbank et al. 2011), may be forming GCs as well (Kravtsov \& Gnedin 2005; Kruijssen 2015). Because these clumps are dense $\left(\sum_{\text {gas }} \sim 10-100 M_{\odot} \mathrm{pc}^{-3}\right)$ and strongly turbulent $\left(\sigma_{\text {gas }} \sim\right.$ $10-100 \mathrm{~km} \mathrm{~s}^{-1}$ ), they are likely to host high-pressure subclumps that can turn into a GC. Based on the assumption that these massive clumps share the gas-phase metallicity of their host galaxy, Shapiro et al. (2010) claim that the number of metal-rich GCs observed in intermediate-mass galaxies with $\sim 10^{10}-10^{11} M_{\odot}$ may be explained by the number of massive clumps, provided that $\sim 700 \mathrm{GCs}$ form per clump and only $\sim 2 \%$ of them survive the two-body relaxation-driven tidal evaporation (Jordán et al. 2007).

On the other hand, the origin of metal-poor GCs has been highly debated. Some authors argue that metal-poor GCs form 
essentially through the same processes as metal-rich GCs in gas-rich, massive galaxies at high redshifts (Kravtsov \& Gnedin 2005; Kruijssen 2015), except that the host galaxy should be less massive to match the lower metallicity of the blue GCs. In a similar context, Elmegreen et al. (2012) propose that $\operatorname{Ly} \alpha$ emitters, which are low-mass, dense, and actively star-forming galaxies (Finkelstein et al. 2007; Gawiser et al. 2007; Pirzkal et al. 2007), can produce as many as the massive $\left(>2 \times 10^{5} M_{\odot}\right)$ blue GCs observed in the local Universe. Alternatively, some metal-poor GCs may have formed at the center of low-mass halos in the young universe, possibly before reionization $(z>6)$ (e.g., Katz \& Ricotti 2013). Peebles (1984) suggested that metal-poor GCs may arise in extended dark halos with $10^{8} M_{\odot}$ when a gas cloud of mass $10^{6} M_{\odot}$ becomes Jeans unstable (see also Fall \& Rees 1985). Bromm \& Clarke (2002) extended the idea by performing three-dimensional hydrodynamic simulations, and speculated that fragmented gas clumps with $10^{5} M_{\odot}$ in the atomic-cooling halos may turn into GCs. Boley et al. (2009) later showed that two simulated clusters of relatively small masses $\left(\sim 5 \times 10^{4} M_{\odot}\right)$ are indeed formed in a minihalo with $5 \times 10^{6} M_{\odot}$ at $z \sim 13$ in their cosmological simulations with star formation and SN feedback. Recently, Trenti et al. (2015) proposed that a merger of two $10^{8} M_{\odot}$ halos that are preenriched by $\mathrm{SNe}$ from nearby (proto-)galaxies may trigger the formation of a metal-poor GC. The main criticism of these dark matter halo (DMH)-based scenarios was that GCs seem to possess no extended halos (Baumgardt et al. 2009; Conroy et al. 2011; Ibata et al. 2013), but it is possible that metal-poor GCs form through several different channels as aforementioned. Also dynamical modeling of mergers of DMHs suggests that tidal stripping can remove a substantial amount of dark matter before stellar components are affected (e.g., Mashchenko \& Sills 2005; Smith et al. 2015), making this scenario still viable.

The DMH-based scenario is barely studied from a baryonic physics viewpoint in a fully cosmological setup, however (see Boley et al. 2009, for an exception). GCs are conventionally known as a simple stellar population (SSP) with homogeneous chemical composition, although more complex features, such as abundance variations (Gratton et al. 2004; Lim et al. 2015) or multiple stellar populations (e.g., Lee et al. 1999; Bedin et al. 2004; Joo \& Lee 2013), are observed. One might speculate that these two well-known features (coeval age and chemical homogeneity) can easily be reproduced if a gas clump with an appropriate mass and metallicity is turned into a cluster instantaneously. But this is a non-trivial problem in a cosmological context. First, cosmic accretion supplies fresh gas to a DMH continuously, possibly resulting in populations with extended star formation histories rather than an SSP. Depending on how rapidly or slowly stellar feedback processes regulate gas collapse and star formation, the mass of a star cluster may vary significantly. Second, if a substantial amount of gas that dominates the gravity of the GC-forming regions is removed by stellar feedback before a self-gravitating star cluster forms, they are likely to expand non-adiabatically, resulting in a diffuse structure (e.g., Pontzen \& Governato 2012). An important question is thus whether a massive $\left(>2 \times 10^{5} M_{\odot}\right)$ and dense structure can ever be formed in a cosmological environment where gas accretion appears to occur slowly and continuously. Third, given that metal enrichment and mixing are unlikely to be homogeneous and instantaneous, the interplay between star formation and feedback may produce chemically inhomogeneous populations of stars. The aim of this paper is to examine these three aspects of GC formation by carrying out a high-resolution zoom-in simulation with realistic stellar feedback processes. Although there could be other formation channels at high redshift (e.g., Kravtsov \& Gnedin 2005; Shapiro et al. 2010; Elmegreen et al. 2012; Kruijssen 2015), we restrict our attention to the scenario based on small DMHs in this work.

\section{NUMERICAL METHOD}

We perform a cosmological radiation hydrodynamic simulation with the adaptive mesh refinement code, RAMSES-RT (Teyssier 2002; Rosdahl \& Teyssier 2015). The initial conditions are generated using MUSIC software (Hahn \& Abel 2011), with the cosmological parameters $\left(\Omega_{\mathrm{m}}=0.288, \quad \Omega_{\Lambda}=0.712, \quad \Omega_{\mathrm{b}}\right.$ $=0.045, \quad H_{0}=69.33 \mathrm{~km} \mathrm{~s}^{-1} \mathrm{Mpc}^{-1}, \quad n_{s}=0.971, \quad$ and $\sigma_{8}$ $=0.830$ ) consistent with the WMAP9 results (Hinshaw et al. 2013). The entire simulation box of $\left(3 \mathrm{Mpch}^{-1}\right)^{3}$ (comoving) is covered with $128^{3}$ root cells. High-resolution dark matter particles of mass $303 M_{\odot}$ are adopted for a zoom-in region of $0.323 \mathrm{Mpc}^{3}$ (comoving), which encompasses two atomiccooling halos with $4 \times 10^{7} M_{\odot}<M_{\text {halo }}<7 \times 10^{7} M_{\odot}$ at $z \sim 10$. The zoom-in region is further refined to attain the maximum resolution of $0.1 \mathrm{pc}$ (physical) if the total mass within a cell exceeds $2424 M_{\odot}$ or if the gas mass in the cell with $n_{\mathrm{H}} \geqslant 0.1 \mathrm{~cm}^{-3}$ exceeds $1.4 M_{\odot}$. We also ensure that the shell formation radius of SN explosions is resolved by three cells (e.g., Kim \& Ostriker 2015), and that above densities of $10 \mathrm{H} / \mathrm{cc}$, the Jeans length is resolved by 32 cells. The Euler equations are solved using the second-order MUSCL scheme with an HLLC Riemann solver, and the Poisson equation is computed using a multi-grid method (Guillet \& Teyssier 2011). For the transport of the three photon groups bracketed by ionization frequencies for $\mathrm{H}_{\mathrm{I}}, \mathrm{He} \mathrm{I}$, and He II, we adopt a GLF solver with a reduced speed of light approximation to reduce the computational costs ( $\hat{c}=10^{-3} c$, Rosdahl et al. 2013, where $c$ is the speed of light.).

Star formation is modeled as a stochastic process (Rasera \& Teyssier 2006), based on a Schmidt law (Schmidt 1959), as $\dot{\rho}_{\text {star }}=\epsilon_{\mathrm{SF}} \rho_{\mathrm{gas}} / t_{\mathrm{ff}}$. Here, $\epsilon_{\mathrm{SF}}$ is the star formation efficiency per local free-fall time $\left(t_{\mathrm{ff}}=\sqrt{3 \pi / 32 G \rho_{\text {gas }}}\right)$, which we take to be $2 \%$ (Kennicutt 1998; Krumholz \& Tan 2007). The sites of star formation are limited to a dense $\left(n_{\mathrm{H}} \geqslant 10^{5} \mathrm{~cm}^{-3}\right)$ and converging gas flow $\left(\boldsymbol{\nabla} \cdot \rho_{\text {gas }} \boldsymbol{v}_{\text {gas }}<0\right)$, where $\boldsymbol{v}_{\text {gas }}$ is the gas velocity. The mass of each star particle $\left(91 M_{\odot}\right)$ is chosen such that it hosts a single SN event for a Kroupa initial mass function (IMF) (Kroupa 2001). ${ }^{5}$ We assume that $21 \%$ of the stellar mass is returned to the surroundings, among which $5 \%$ in mass is the newly synthesized metals. This corresponds to a metal yield of $\approx 0.01$ for an SSP with $1 M_{\odot}$.

We assume the initial metallicity of the simulation to be zero. Radiative gas cooling is computed by following the nonequilibrium chemistry of $\mathrm{HI}_{\mathrm{I}}, \mathrm{H}_{\mathrm{II}}, \mathrm{He} \mathrm{I}, \mathrm{He}$ II, $\mathrm{He}$ III, and $e^{-}$,

\footnotetext{
5 This neglects the fact that one would require $\sim 10^{3}-10^{4} M_{\odot}$ to fully sample the IMF (see Kroupa et al. 2013, for a recent review). Choosing such a small mass for the star particle may lead to SNe exploding earlier than the realistic case and may over-predict the initial chemical enrichment. However, given that a large amount $\left(10^{6} M_{\odot}\right)$ of star-forming gas collapses on a very short timescale $\left(t_{\mathrm{ff}} \lesssim 0.1 \mathrm{Myr}\right)$ and stars amounting to $10^{4} M_{\odot}$ form quickly $(\sim 1 \mathrm{Myr})$ in our simulations, we do not expect that fully sampling the IMF would significantly affect our main results on the stellar mass and metallicity distributions of the simulated clusters
} 


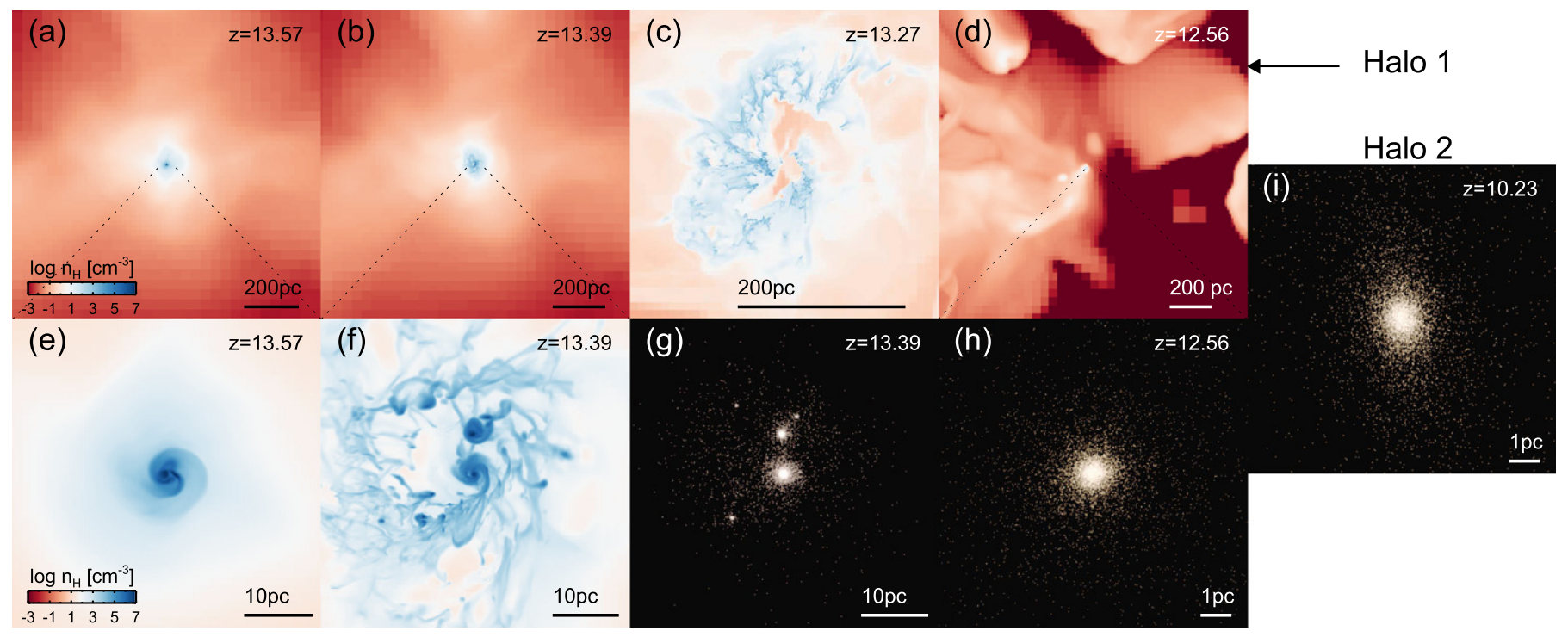

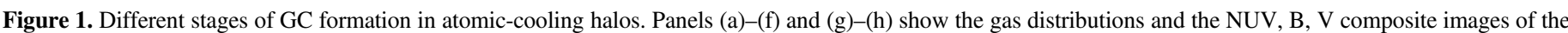

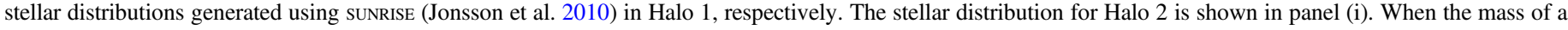

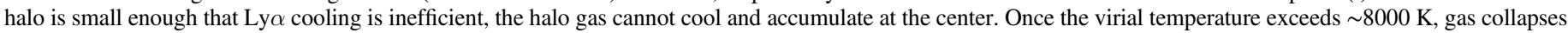

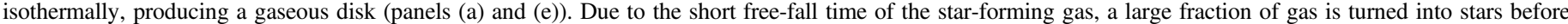

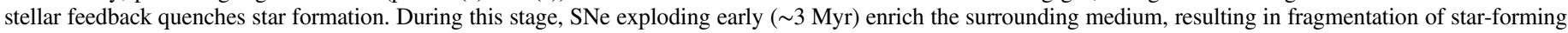

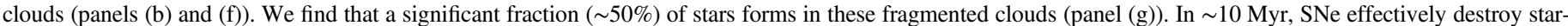

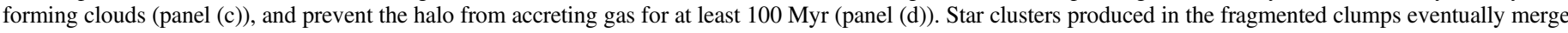
into a single massive, compact cluster (panel (h)).

coupled with the radiation. We also include metal cooling under the assumption of collisional equilibrium for $T \gtrsim 10^{4} \mathrm{~K}$ (Sutherland \& Dopita 1993). Gas can cool down further with the metal fine-structure transitions (Rosen \& Bregman 1995). Since our simulation explicitly follows the local ionizing radiation, we do not use the uniform ultraviolet background field.

Our simulation includes three different forms of stellar feedback. First, ionizing photons from massive stars can heat up gas to $\approx 2 \times 10^{4} \mathrm{~K}$ through photo-ionization, which subsequently reduces the gas density by over-pressurizing the surrounding medium. Second, absorption of ionizing photons from an SSP with $1 M_{\odot}$ transfers the radial momentum of $\sim 40 \mathrm{~km} \mathrm{~s}^{-1} M_{\odot}$ to the surroundings. This is done by imparting momentum from ionizing radiation continuously for a given stellar metallicity and age, based on Leitherer et al. (1999). Finally, SN explosions are modeled by injecting the radial momentum calculated according to the stage of the SedovTaylor blast wave (Kimm \& Cen 2014). We also take into account the continuous spectrum of the lifetime of a massive star (from 3 to $40 \mathrm{Myr}$ ) (Kimm et al. 2015). The simulations are run to $z=10.2$, by which two simulated GCs formed in atomiccooling halos have become quiescent.

\section{RESULTS}

In Figure 1, we present the evolution of gas structures and the formation of two GC systems in atomic-cooling DMHs $\left(M_{\text {halo }} \sim 4-7 \times 10^{7} M_{\odot}\right)$ at high redshift $(z \gtrsim 10)$. This may be summarized by five different evolutionary stages. (1) When the halo mass is so small that its virial temperature is lower than $\sim 8000 \mathrm{~K}$, the gas in the mini-halo of primordial composition cannot cool through $\operatorname{Ly} \alpha$ emission and, thus, remains rather diffuse $\left(n_{\mathrm{H}} \lesssim 100 \mathrm{~cm}^{-3}\right)$ (adiabatic phase). (2) Once a $\mathrm{DMH}$ becomes more massive than the transition mass
$M_{\text {halo,tr }}=4 \times 10^{7}\left(\frac{1+z}{11}\right)^{-1.5} M_{\odot}$ through smooth accretion or halo mergers, the virial temperature of the halo exceeds $\sim 8000 \mathrm{~K}$ and the gas in the central region of the halo collapses nearly isothermally with the aid of strong Ly $\alpha$ cooling (panels (a) and (e)). Because the halo gas carries angular momentum generated by the large-scale tidal torque, the collapse leads to the formation of a dense, disk-like structure (e.g., Regan \& Haehnelt 2009) (isothermal cooling phase). (3) As the density of the disk becomes very high $\left(n_{\mathrm{H}} \gtrsim 10^{6} \mathrm{~cm}^{-3}\right)$, its gas is turned efficiently into stars on a very short timescale $(\lesssim 10 \mathrm{Myr})$. During this stage, early SNe $(t \sim 3 \mathrm{Myr})$ provide newly synthesized metals to the surrounding medium. The metals enhance radiative cooling in the star-forming disk, resulting in gas fragmentation (panels (b) and (f). We find that roughly half of the stars (56\% and $50 \%$, respectively) in the simulated GCs form in the fragmented clumps (panel(g)) (selfenrichment and fragmentation phase). (4) A large number of SN explosions $\left(\sim 10^{4}\right)$ ensue, clearing out the star-forming clouds and gas in the central region of the halo (panel (c)) (outflow phase). (5) Subsequent star formation is quenched for at least one hundred Myr, and star clusters at the center of the halo quickly merge into a single cluster. Then, dynamical interactions of stars inside each cluster are likely to govern the structural evolution from this point (e.g., Gao et al. 1991).

We find that the resulting dense clusters in the two halos share two important properties with the local GC populations. First, the mass of the simulated GCs $\left(6 \times 10^{5} M_{\odot}\right.$ for both halos) is comparable to the local GCs (Harris 1996; Strader et al. 2011). If we assume that the stars in these systems are unaffected by tidal stripping and simply evolve to $z=0$, their $V$-band magnitudes would also be consistent with observations (Figure 2). Here we compute the $V$ band flux assuming a Kroupa IMF with a low- (high-)mass cut-off of 0.1 (100) $M_{\odot}$ (Leitherer et al. 1999). Second, the simulated GCs are 


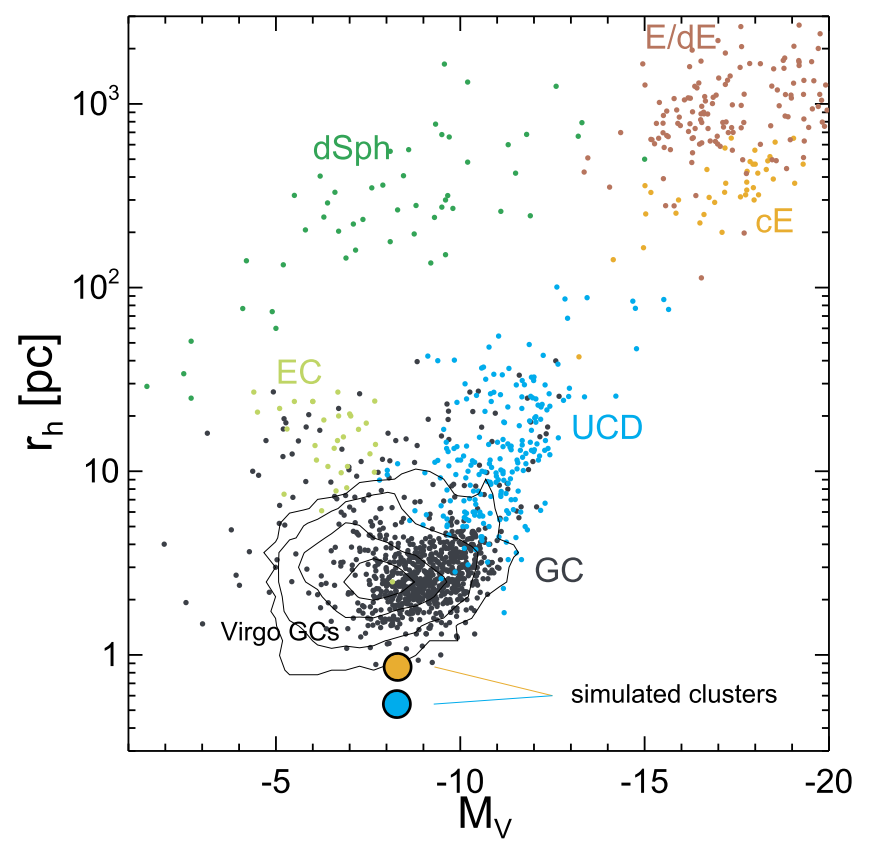

Figure 2. Comparison of half-light radii and $V$-band magnitudes between the two simulated GCs (filled circles with the black envelope) and the observations compiled by Brodie et al. (2011). Data points are color-coded according to their structural types (GC: globular clusters, UCD: ultracompact dwarves, EC: extended clusters, dSph: dwarf spheroidal, dE: dwarf ellipticals, cE: compact ellipticals). Also included as black contours is the distribution $(10 \%, 68 \%$, 95\%, 99.5\%) of the GCs from the ACS Virgo Cluster Survey (Jordán et al. 2009). We use the empirical color transformation of the GCs to convert from $g$ and $z$ bands to $V$ band (Forbes et al. 2013). The size of the simulated clusters is measured when a single massive cluster is formed after mergers of clusters in the disk. The $V$-band magnitudes of the simulated GCs are calculated assuming that member stars evolve to $z=0$ without any loss due to dynamical interactions.

found to be very compact. When mergers of several clusters are completed and form a single massive cluster, the threedimensional half-light radius is 0.54 and $0.86 \mathrm{pc}$, respectively. Although the size of the simulated clusters is approximately a factor of 3-5 smaller than the observational estimates (e.g., Harris 1996; Jordán et al. 2009; Brodie et al. 2011), we note that subsequent dynamical evolutions through binary interactions (e.g., Gao et al. 1991) or mass loss are likely to increase the size of the systems. In a similar context, Baumgardt et al. (2010) suggests that the initial size of the local compact GCs is likely to be less than $1 \mathrm{pc}$ (cf. Shin et al. 2013). ${ }^{6}$ It is also worth mentioning that each simulated cluster is formed in a single burst of star formation ( $\lesssim 10 \mathrm{Myr}$ ) (Figure 3), roughly consistent with the fact that a GC is essentially an SSP. ${ }^{7}$ Note

\footnotetext{
6 Note that the accurate determination of the initial size of GCs will require the inclusion tidal shocks due to giant molecular clouds, which is not taken into consideration in Baumgardt et al. (2010).

7 We note, however, that the simulated clusters would have formed on a timescale shorter than $\sim 10 \mathrm{Myr}$ if the effect of radiation feedback was stronger. Due to the lack of cooling agents in our primordial halos, the temperature of the star-forming gas does not drop far below $\sim 10^{4} \mathrm{~K}$, and the external pressure around the star-forming cloud is over-estimated. As a result, $\mathrm{H}$ II bubbles are confined by the surroundings and cannot drive outflows in our simulation. Including relevant cooling processes by molecular hydrogen and metals produced by Pop III/II stars will decrease the temperature of the star-forming regions and result in an enhanced photoionization feedback before $\mathrm{SNe}$ explode. This is likely to reduce the star formation timescale, which will be more compatible with the age spreads found in the local young massive clusters $(\lesssim 3 \mathrm{Myr})$.
}

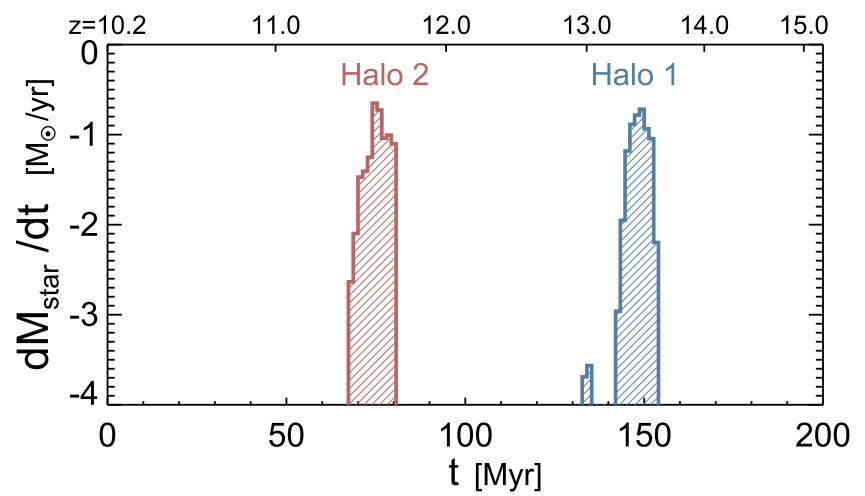

Figure 3. Star formation histories of the two simulated GCs. Stars form very efficiently within $\lesssim 10 \mathrm{Myr}$ before SNe effectively destroy star-forming clouds and suppress gas accretion. The resulting star clusters remain as an SSP within the timescale of our simulation.

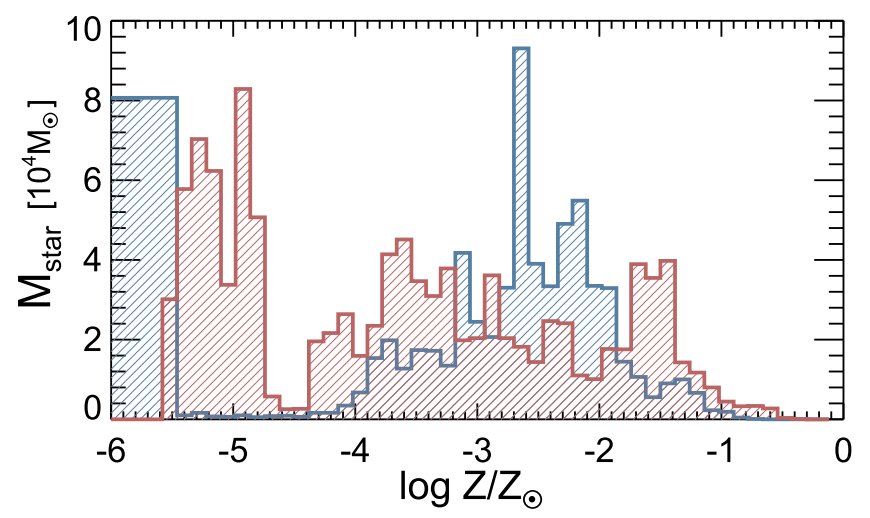

Figure 4. Metallicity distributions of stars in the two simulated GCs. Different colors denote different GCs, as in Figure 3. The mean metallicities of the stellar systems are 0.006 and $0.012 Z_{\odot}$, respectively. Metallicities lower than $10^{-6} Z_{\odot}$ are shifted to $10^{-6} Z_{\odot}$ in Halo 1 . Note that there are no stars with zero metallicity in Halo 2, as they are pre-enriched by the SN ejecta from Halo 1. Although a fraction of stars is quickly enriched to $0.1 Z_{\odot}$, we find that there is a wide spread in metallicities in contrast to observations.

that such a sharp truncation in star formation is driven by efficient SN feedback.

Although the predicted size and mass are comparable with their local counterparts, we find that the simulated GCs show a wide spread in metallicities. The mass-weighted mean metallicity of the two GC candidates $\left(0.006\right.$ and $\left.0.012 Z_{\odot}\right)$ is close to that of the local metal-poor populations, but the metallicity of individual star particles is found to range from zero to a tenth of Solar (Figure 4). Such a large dispersion is not unexpected because our simulations start from zero metallicity. Yet it is interesting to note that the fraction of the extremely metal-poor population $\left(Z \lesssim 10^{-3} Z_{\odot}\right)$ is significant $(50 \%-$ $60 \%$ ) in contrast to the standard interpretation that a GC is rather a chemically homogeneous system (Harris 1996). This can be attributed to the fact that, in simulations, a large fraction of gas is converted into stars before the first $\mathrm{SN}$ emerges and pollutes the star-forming gas. Moreover, as metals from $\mathrm{SNe}$ are dispersed locally and some of $\mathrm{SNe}$ exploding on the outskirt of star-forming clouds can simply escape from them, we find that the metal enrichment occurs in a very inhomogeneous fashion. For example, Figure 5 shows that the second massive star-forming cloud (upper giant cloud in the first panel) is at least an order of magnitude more metal-poor than the central gas clump. However, it should be noted that the 


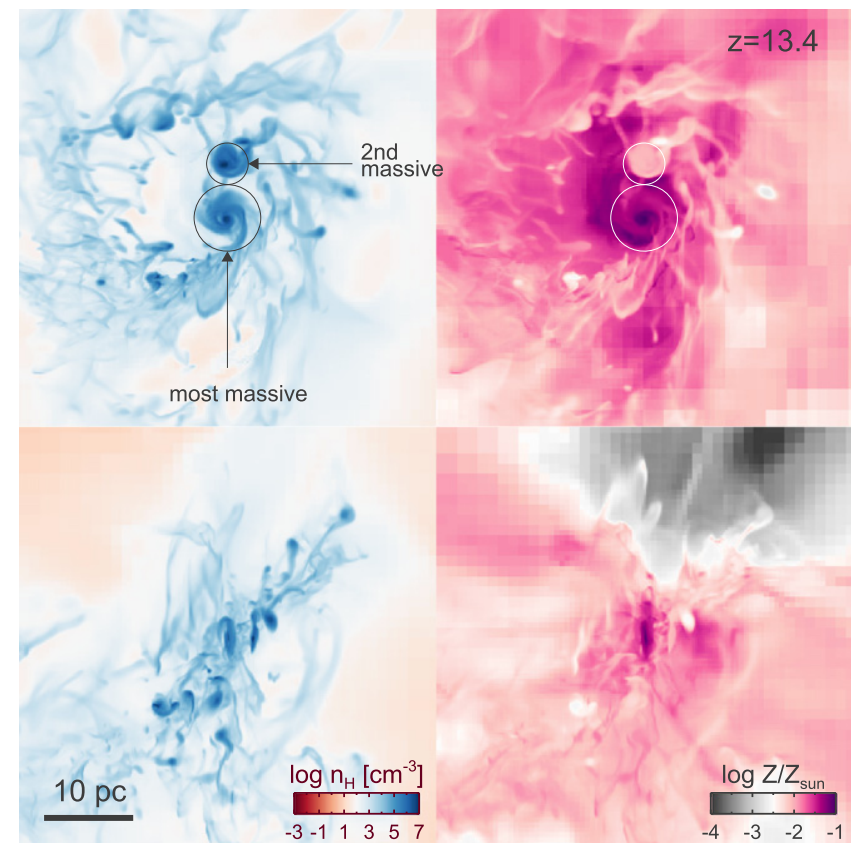

Figure 5. Inhomogeneous distributions of metals during the self-enrichment by SN explosions. The mass-weighted density and metallicity distributions are shown in left and right panels, respectively. Top and bottom panels are different projections $(x, z)$. forming clumps $\left(n_{\mathrm{H}} \geqslant 10^{5} \mathrm{~cm}^{-3}\right)$ exhibit a wide range of metallicities. The non-uniform metal enrichment appears inevitable in the scenario where GCs form from pristine halo gas, posing a challenge to GC formation theory.

metallicity distributions may not necessarily rule out the DMHbased scenario, because pre-enrichment by Pop III and II stars can reduce the metallicity spread by effectively regulating the self-enrichment via radiation feedback. It is also possible that the inclusion of turbulent metal mixing (e.g., Shen et al. 2010) that is not fully resolved in this study would suppress the formation of high- and low-metallicity stars. We discuss a role of pre-enrichment by the first generation stars and metal mixing as a potential solution to this problem in the next section.

\section{DISCUSSION AND CONCLUSIONS}

Using cosmological radiation hydrodynamic simulations, we show that a massive and compact star cluster can form at the center of a DMH during the transition from molecular-cooling to atomic-cooling halos, provided that gas is primordial and atomic. Because gas collapse occurs isothermally under such conditions, a large fraction of the gaseous disk with very high density turns into stars before SNe disrupt the star-forming clouds. During this stage, the gaseous disk is fragmented and stars form in each clump due to self-enrichment from early $\mathrm{SNe}$ commencing at $3 \mathrm{Myr}$, unlike the picture of black hole seed formation in which gas simply collapses onto au scales (e.g., Choi et al. 2013). The resulting star clusters are found to have similar physical properties (size and mass) to the local GC populations, except for metallicity spread and the possibly related star formation timescale.

The most critical assumption in this scenario is that gas is primordial and atomic so that cooling due to molecular hydrogen and subsequent star formation does not prevent a large amount of gas from accumulating in the central region of the halo to form a GC. This may be achieved by having a strong Lyman-Werner background radiation that photo- dissociates molecular hydrogen (e.g., Omukai 2001). However, we argue that even with cooling due to molecular hydrogen or metals produced by neighboring galaxies, it may still be possible to form a dense cluster, provided that Pop III or Pop II populations effectively regulate gas accretion. If gas is allowed to collapse in mini-halos via molecular hydrogen cooling and form Pop III stars (Greif et al. 2010), SN explosions and radiation feedback may expel a large fraction of the halo gas, suppressing star formation. If stellar feedback is energetic enough, subsequent major gas collapse is likely to occur when a large amount of gas is available and radiative cooling becomes significant, i.e., when the halo mass becomes close to the atomic-cooling regime. Even in the case where the recovery timescale of gas accretion is short due to weak feedback from Pop III stars, the first generation of Pop II stars can in principle blow away the interstellar medium without constituting the GC population if the ensuing collapse of gas does not occur exactly inside the first Pop II stars (T. Kimm et al. 2016, in preparation). Given that Pop III stars can pre-enrich the halo gas to $Z \sim 10^{-3}-10^{-2} Z_{\odot}$ (Boley et al. 2009; Greif et al. 2010; Ritter et al. 2012) and halos with a few times $10^{7} M_{\odot}$ are likely to produce $\sim 100 M_{\odot}$ of metals (e.g., Wise et al. 2014), it can also help to alleviate the metallicity spread found in our simulation, possibly explaining the helium enhancement observed in some GCs (Choi \& Yi 2007).

We note that the DMH-based scenario may be able to accommodate the observed number of blue GCs if a significant fraction of DMHs forms a GC during the transition from molecular-cooling to atomic-cooling regime. To estimate the maximum frequency, we generate halo merger trees for the volume of $\left(100 \mathrm{Mpc} \mathrm{h}^{-1}\right)^{3}$ using the Monte Carlo algorithm based on the extended Press Schechter theory (Parkinson et al. 2008). We adopt the same cosmological parameters as described in Section 2 and a mass resolution of $M_{\text {res }}=5 \times 10^{6} M_{\odot}$. Then the number of halos that first cross the transition mass $\left(M_{\text {halo }}=4 \times 10^{7}\left(\frac{1+z}{11}\right)^{-1.5} M_{\odot}\right)$ is computed throughout the merger history in each halo. We find that the resulting frequency is $N_{\mathrm{GC}, \max } \approx 650 \pm 20 \times$ $\left(M_{\text {halo }} / 10^{12} M_{\odot}\right)$. Thus, for a Milky Way hosting halo of mass $10^{12} M_{\odot}$, the DMH-based formation scenario can predict the number of GCs up to six times more than the number of the local metal-poor population (e.g., Harris 1996) or an order of magnitude larger stellar masses associated with GC systems per DMH mass (Spitler \& Forbes 2009; Durrell et al. 2014; Hudson et al. 2014). The large number of GCs may seem inconsistent with the local values, but it should be noted that the actual abundance at $z=0$ is likely to be lower than the maximum estimate for several reasons. It is known that the GC system can be reduced or disrupted by two-body relaxationdriven, tidally limited evaporation (Fall \& Zhang 2001; Vesperini 2001), disk and bulge shocks (Gnedin \& Ostriker 1997), and tidal shocks by giant molecular clouds in the disk (Kruijssen 2012, 2015). It is also possible that the system ends up as a nuclear star cluster instead of a GC, given that it is embedded in the central region of a DMH. More importantly, not all of the DMHs may host a GC if gas accretion takes place rather continuously due to the inefficient explosion of the first generation stars and form a dwarf galaxy. In this regard, we argue that the large maximum frequency of GCs simply indicates that the DMH-based scenario can easily accommodate the abundance of local blue GCs. 
Finally, it should be mentioned that the DMH-based scenario may be ruled out if overall metal enrichment from the first generation stars (i.e., Pop III and Pop II) or external stellar sources (Trenti et al. 2015) is not sufficient enough to reach the typical metallicity of metal-poor GCs $\left(Z \sim 0.02 Z_{\odot}\right)$. As shown in Figures 4 and 5, the self-enrichment by stars in the simulated GCs is highly inhomogeneous and leads to a wide stellar metallicity distribution of $10^{-5} \lesssim Z / Z_{\odot} \lesssim 10^{-1}$, in contradiction to the observed metallicity spread (Gratton et al. 2012). Part of the large spread may be caused by the neglect of turbulent metal mixing that is not fully captured in our simulation due to limited resolution. The inclusion of explicit turbulent metal mixing (e.g., Shen et al. 2010) is likely to spread out the metal from highly enriched regions $\left(Z \gg 0.02 Z_{\odot}\right)$ and increase the metallicity in metal-poor regions. However, the mixing alone may not be able to solve the metallicity problem, given that low-metallicity stars would still form before the first SNe explode and enrich the interstellar medium. Instead, we note that pre-enrichment by Pop III and II stars can significantly reduce the metallicity spread by removing the low-metallicity tail. This pre-enrichment, along with the cooling by molecular hydrogen, can also effectively suppress the formation of high-metallicity stars for the following reason. Due to the lack of cooling agents in our primordial halos, we find that the temperature of star-forming gas does not drop far below $8000 \mathrm{~K}$ during the early stage of star formation, making the external gas pressure in the starforming regions too high. This leads to an underestimation of the effect of photoionization feedback, because the $\mathrm{H}$ II region is no longer an over-pressurized bubble and cannot generate enough momentum to disperse gas clouds on a very short time scale of a few Myr (Walch et al. 2012; Dale et al. 2014). However, including the relevant cooling processes by metal and molecular hydrogen will lower the temperature of the starforming region, and the inner pressure from $\mathrm{H}$ II regions will be able to drive outflows and suppress star formation before newly synthesized metals from SNe II ejecta is recycled to starforming clumps. Thus, the metallicity problem may be solved by taking into account Pop III stars and relevant cooling processes. In this case, the self-enrichment process will be limited accordingly, and one would require the pre-enrichment of metals that closely match those of local blue GCs. If a wide range of pre-enrichment by Pop III stars is real ( $Z \sim 10^{-3}-10^{-2} Z_{\odot}$, Boley et al. 2009; Greif et al. 2010; Ritter et al. 2012) and only a fraction of the metal ejecta from the first Pop II stars is recycled due to outflows, an important question would then be why GC formation is efficient only in regions with $Z \gtrsim 0.02 Z_{\odot}$. Future studies with Pop III stars and fullblown chemistry will be useful to more accurately assess the formation scenario of GCs at the center of DMHs.

We are indebted to an anonymous referee for a constructive and thorough report that improved the presentation of this work. We thank Kengo Tomida for useful discussions, and Romain Teyssier for making his code RAMSES publicly available. Computing resources were in part provided by the NASA High-end Computing (HEC) Program through the NASA Advanced Supercomputing (NAS) Division at Ames Research Center and in part by the Horizon-UK program through DiRAC-2 facilities. The research is supported in part by NSF grant AST-1108700 and NASA grant NNX12AF91G and in part by the ERC Advanced Grant 320596 "The
Emergence of Structure during the epoch of Reionization." JR was funded by the European Research Council under the European Unions Seventh Framework Programme (FP7/20072013)/ERC Grant agreement 278594-GasAroundGalaxies, and the Marie Curie Training Network CosmoComp (PITN-GA2009-238356). SKY acknowledges support from the Korean National Research Foundation (Doyak 2014003730).

\section{REFERENCES}

Bastian, N., Trancho, G., Konstantopoulos, I. S., \& Miller, B. W. 2009, ApJ, 701, 607

Baumgardt, H., Côté, P., Hilker, M., et al. 2009, MNRAS, 396, 2051

Baumgardt, H., Parmentier, G., Gieles, M., \& Vesperini, E. 2010, MNRAS, 401, 1832

Bedin, L. R., Piotto, G., Anderson, J., et al. 2004, ApJL, 605, L125

Boley, A. C., Lake, G., Read, J., \& Teyssier, R. 2009, ApJL, 706, L192

Brodie, J. P., Romanowsky, A. J., Strader, J., \& Forbes, D. A. 2011, AJ, 142,199

Bromm, V., \& Clarke, C. J. 2002, ApJL, 566, L1

Choi, E., \& Yi, S. K. 2007, MNRAS, 375, L1

Choi, J.-H., Shlosman, I., \& Begelman, M. C. 2013, ApJ, 774, 149

Conroy, C., Loeb, A., \& Spergel, D. N. 2011, ApJ, 741, 72

Dale, J. E., Ngoumou, J., Ercolano, B., \& Bonnell, I. A. 2014, MNRAS, 442, 694

Dubois, Y., Pichon, C., Welker, C., et al. 2014, MNRAS, 444, 1453

Durrell, P. R., Côté, P., Peng, E. W., et al. 2014, ApJ, 794, 103

Elmegreen, B. G., \& Efremov, Y. N. 1997, ApJ, 480, 235

Elmegreen, B. G., Elmegreen, D. M., Fernandez, M. X., \& Lemonias, J. J. 2009, ApJ, 692, 12

Elmegreen, B. G., Malhotra, S., \& Rhoads, J. 2012, ApJ, 757, 9

Fall, S. M., \& Rees, M. J. 1985, ApJ, 298, 18

Fall, S. M., \& Zhang, Q. 2001, ApJ, 561, 751

Finkelstein, S. L., Rhoads, J. E., Malhotra, S., Pirzkal, N., \& Wang, J. 2007, ApJ, 660, 1023

Forbes, D. A., Pota, V., Usher, C., et al. 2013, MNRAS, 435, L6

Gao, B., Goodman, J., Cohn, H., \& Murphy, B. 1991, ApJ, 370, 567

Gawiser, E., Francke, H., Lai, K., et al. 2007, ApJ, 671, 278

Genzel, R., Burkert, A., Bouché, N., et al. 2008, ApJ, 687, 59

Gnedin, O. Y., \& Ostriker, J. P. 1997, ApJ, 474, 223

Governato, F., Willman, B., Mayer, L., et al. 2007, MNRAS, 374, 1479

Gratton, R., Sneden, C., \& Carretta, E. 2004, ARA\&A, 42, 385

Gratton, R. G., Carretta, E., \& Bragaglia, A. 2012, A\&ARv, 20, 50

Greif, T. H., Glover, S. C. O., Bromm, V., \& Klessen, R. S. 2010, ApJ, 716,510

Guillet, T., \& Teyssier, R. 2011, JCoPh, 230, 4756

Hahn, O., \& Abel, T. 2011, MNRAS, 415, 2101

Harris, W. E. 1996, AJ, 112, 1487

Hinshaw, G., Larson, D., Komatsu, E., et al. 2013, ApJS, 208, 19

Hudson, M. J., Harris, G. L., \& Harris, W. E. 2014, ApJL, 787, L5

Ibata, R., Nipoti, C., Sollima, A., et al. 2013, MNRAS, 428, 3648

Jonsson, P., Groves, B. A., \& Cox, T. J. 2010, MNRAS, 403, 17

Joo, S.-J., \& Lee, Y.-W. 2013, ApJ, 762, 36

Jordán, A., McLaughlin, D. E., Côté, P., et al. 2007, ApJS, 171, 101

Jordán, A., Peng, E. W., Blakeslee, J. P., et al. 2009, ApJS, 180, 54

Katz, H., \& Ricotti, M. 2013, MNRAS, 432, 3250

Kennicutt, R. C., Jr. 1998, ApJ, 498, 541

Kim, C.-G., \& Ostriker, E. C. 2015, ApJ, 802, 99

Kimm, T., \& Cen, R. 2014, ApJ, 788, 121

Kimm, T., Cen, R., Devriendt, J., Dubois, Y., \& Slyz, A. 2015, MNRAS, 451, 2900

Kravtsov, A. V., \& Gnedin, O. Y. 2005, ApJ, 623, 650

Kroupa, P. 2001, MNRAS, 322, 231

Kroupa, P., Weidner, C., Pflamm-Altenburg, J., et al. 2013, in The Stellar and Sub-Stellar Initial Mass Function of Simple and Composite Populations, ed.

T. D. Oswalt, \& G. Gilmore (Dordrecht: Springer), 115

Kruijssen, J. M. D. 2012, MNRAS, 426, 3008

Kruijssen, J. M. D. 2015, MNRAS, 454, 1658

Krumholz, M. R., \& Tan, J. C. 2007, ApJ, 654, 304

Lee, Y.-W., Joo, J.-M., Sohn, Y.-J., et al. 1999, Natur, 402, 55

Leitherer, C., Schaerer, D., Goldader, J. D., et al. 1999, ApJS, 123, 3

Lim, D., Han, S.-I., Lee, Y.-W., et al. 2015, ApJS, 216, 19

Mashchenko, S., \& Sills, A. 2005, ApJ, 619, 258

Mihos, J. C., \& Hernquist, L. 1996, ApJ, 464, 641 
Naab, T., Khochfar, S., \& Burkert, A. 2006, ApJL, 636, L81

Omukai, K. 2001, ApJ, 546, 635

Parkinson, H., Cole, S., \& Helly, J. 2008, MNRAS, 383, 557

Peebles, P. J. E. 1984, ApJ, 277, 470

Pichon, C., Pogosyan, D., Kimm, T., et al. 2011, MNRAS, 418, 2493

Pirzkal, N., Malhotra, S., Rhoads, J. E., \& Xu, C. 2007, ApJ, 667, 49

Pontzen, A., \& Governato, F. 2012, MNRAS, 421, 3464

Rasera, Y., \& Teyssier, R. 2006, A\&A, 445, 1

Regan, J. A., \& Haehnelt, M. G. 2009, MNRAS, 396, 343

Renaud, F., Bournaud, F., \& Duc, P.-A. 2015, MNRAS, 446, 2038

Ritter, J. S., Safranek-Shrader, C., Gnat, O., Milosavljević, M., \& Bromm, V. 2012, ApJ, 761, 56

Rosdahl, J., Blaizot, J., Aubert, D., Stranex, T., \& Teyssier, R. 2013, MNRAS, 436, 2188

Rosdahl, J., \& Teyssier, R. 2015, MNRAS, 449, 4380

Rosen, A., \& Bregman, J. N. 1995, ApJ, 440, 634

Sales, L. V., Marinacci, F., Springel, V., \& Petkova, M. 2014, MNRAS, 439, 2990

Schmidt, M. 1959, ApJ, 129, 243

Schweizer, F., Miller, B. W., Whitmore, B. C., \& Fall, S. M. 1996, AJ, 112,1839
Shapiro, K. L., Genzel, R., \& Förster Schreiber, N. M. 2010, MNRAS, 403, L36

Shen, S., Wadsley, J., \& Stinson, G. 2010, MNRAS, 407, 1581

Shin, J., Kim, S. S., Yoon, S.-J., \& Kim, J. 2013, ApJ, 762, 135

Smith, R., Sánchez-Janssen, R., Beasley, M. A., et al. 2015, MNRAS, 454, 2502

Spitler, L. R., \& Forbes, D. A. 2009, MNRAS, 392, L1

Strader, J., Caldwell, N., \& Seth, A. C. 2011, AJ, 142, 8

Sutherland, R. S., \& Dopita, M. A. 1993, ApJS, 88, 253

Swinbank, A. M., Papadopoulos, P. P., Cox, P., et al. 2011, ApJ, 742, 11

Teyssier, R. 2002, A\&A, 385, 337

Teyssier, R., Chapon, D., \& Bournaud, F. 2010, ApJL, 720, L149

Tolstoy, E., Hill, V., \& Tosi, M. 2009, ARA\&A, 47, 371

Toomre, A., \& Toomre, J. 1972, ApJ, 178, 623

Trenti, M., Padoan, P., \& Jimenez, R. 2015, ApJL, 808, L35

Vesperini, E. 2001, MNRAS, 322, 247

Walch, S. K., Whitworth, A. P., Bisbas, T., Wünsch, R., \& Hubber, D. 2012, MNRAS, 427, 625

Whitmore, B. C., \& Schweizer, F. 1995, AJ, 109, 960

Wise, J. H., Demchenko, V. G., Halicek, M. T., et al. 2014, MNRAS, 442,2560 